\title{
Mitochondrial dysfunctions and neurodegenerative diseases: a mini-review
}

\begin{abstract}
Mitochondrial dysfunction is estimated to be the primary reason involved in different types of neurodegenerative disorders as mitochondria is suggested to be important in the production of reactive oxygen species. Recently, several evidences have emerged out for impaired mitochondrial structures and functions viz. shape, size, fission-fusion, distribution, movement etc. in neurodegenerative diseases especially with Alzheimer's disease, Parkinson's disease and Huntington's disease. Therefore, apart from looking neurodegenerative diseases on the whole, a detailed understanding of the functioning of mitochondria and their role in degeneration would pave new options for the therapy of agerelated neurodegenerative diseases
\end{abstract}

Keywords: mitochondrial dysfunction, neurodegenerative disorders, parkinson's disease, huntington's disease, and alzheimer's disease
Volume 10 Issue 4 - 202I

\author{
Ratan Kumari, Nikhila Shekhar, Sakshi Tyagi, \\ Ajit Kumar Thakur \\ School of Pharmaceutical Sciences, Delhi Pharmaceutical \\ Sciences and Research University, New Delhi-I 10 017, India
}

\author{
Correspondence: Dr.Ajit Kumar Thakur,Assistant Professor \\ of Pharmacology, School of Pharmaceutical Sciences, Delhi \\ Pharmaceutical Sciences and Research University, Pushp Vihar \\ Sector-III, M.B. Road, New Delhi-I I 0 0I7, India, \\ Tel 9I-I I-2955348I, Fax 91-I I-29554503 \\ Email ajit.thakur@dpsru.edu.in
}

Received: August 05, 2021 | Published: August 16, 2021

\section{Introduction}

Mitochondria, sometimes known as "the power house of the cell," are the most distinct and important cell organelles in eukaryotes. They create energy in the form of adenosine triphosphate (ATP) by oxidative metabolism of nutrients, which involves two key stages, firstly, oxidation of $\mathrm{NADH}$ or FADH2 generated during glycolysis, TCA cycle, or oxidation of fatty acids, and secondly, oxidative phosphorylation that generate ATP. ${ }^{1}$ In the nervous system, mitochondria -generated ATP is required to establish appropriate electrochemical gradients and reliable synaptic transmission. ${ }^{2}$ When there is a decrease in the production of these ATP or a loss in electron transport chain efficiency due to aging or any chronic illness, mitochondrial dysfunction occurs.

A vast array of neurodegenerative diseases, including Alzheimer's, Parkinson's, Huntington's, amyotrophic lateral sclerosis, and Friedreich's ataxia and autoimmune diseases, such as multiple sclerosis, systemic lupus erythematosus, and type 1 diabetes, autism spectrum disorders, schizophrenia, and bipolar and mood disorders are examples of neurobehavioral and psychiatric illnesses which are characterized by mitochondrial dysfunction. ${ }^{3}$ Retrograde signalling initiated by dysfunctional mitochondria can affect cell morphology and function by causing global alterations in gene expression. This is usually attributed to a disruption of essential mitochondrial processes such as ATP production, metabolic integration, calcium homeostasis, and apoptosis control. ${ }^{4}$

\section{Mitochondrial dysfunction and neurodegenerative diseases}

Mitochondrial dysfunction is estimated to be the primary reason involved in different types of neurodegenerative disorders as mitochondria is suggested to be important in the production of reactive oxygen species (ROS). Figure 1 summarised the cellular events in neurodegenerative disorders particularly at the level of mitochondria. High concentrations of reactive oxygen species (ROS) start a chain reaction that leads to non-apoptotic cell death. ${ }^{5}$ Mitochondria regulate cell death and play a key role in neurodegeneration with the addition of mutated conditions of mitochondrial DNA and oxidative stress. This combinational condition may lead to aging, which is a favourable condition for neurodegenerative disorder. Thus, therapies targeting basic mitochondrial processes, such as energy metabolism or freeradical generation, or specific interactions of disease-related proteins with mitochondria, hold great promise. ${ }^{6}$ Recently, many evidences have emerged for impaired mitochondrial structures and functions viz. shape, size, fission-fusion, distribution, movement etc. in neurodegenerative diseases such as Alzheimer's disease, Parkinson's disease and Huntington's disease. ${ }^{7}$

\section{Alzheimer's disease}

Alzheimer's disease (AD) is a neurodegenerative illness characterized by a neuronal build-up of aberrant tau proteins and amyloid plaques in afflicted brain areas. Although the pathophysiology of Alzheimer's disease has been linked to a build-up of brain amyloid(A) peptide and hyperphosphorylated tau protein, the exact cause of the disease is unknown. Reduced metabolism, disruption of $\mathrm{Ca} 2+$ homeostasis, and higher amounts of reactive oxygen species, lipid peroxidation, and apoptosis have all been found as early events in the etiology of Alzheimer's disease. ${ }^{8}$ Apart from these, damaged mitochondria are thought to play a key role in the pathophysiology of Alzheimer's disease, according to a significant body of research. It is thought that a healthy mitochondrial pool not only promotes neuronal activity by providing enough energy and other mitochondrial functions but also protects neurons by reducing mitochondrial-related oxidative damage. ${ }^{9}$ The first molecules that enter the mitochondrial electron transport chain (ETC) are nicotinamide dinucleotide (NADH). The ETC is made up of a series of reducing agents grouped into four primary protein-metal complexes (I-IV) that use electron transport to produce ATP. ATP synthase, also known as complex V, is another important protein involved in oxidative phosphorylation. As a result of electron flux via the ETC, Complex V performs ATP production. The process of oxidative phosphorylation involves the coupling of electron transport from NADH to molecular oxygen to produce ATP. ${ }^{10}$ 


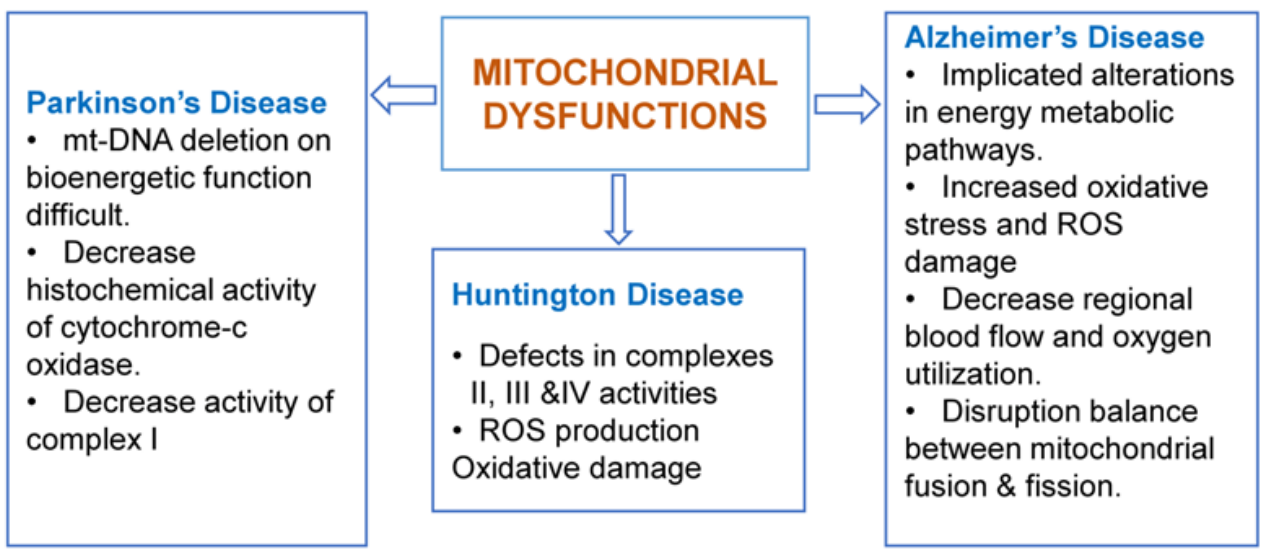

Figure I Mitochondrial dysfunction associated with neurodegenerative diseases.

\section{Parkinson's disease}

Parkinson's disease (PD) is the second most common neurological illness. Around $2 \%$ of the population over the age of 60 is affected by the disease. Parkinson's disease (PD) is characterised by the death of dopaminergic neurons in the substantia nigra in a preferential manner. The disease's clinical hallmarks include the loss of dopaminergic neurons in the substantia nigra and the appearance of Lewy bodies comprised of a-synuclein. Several theories for the pathogenesis of Parkinson's disease have been suggested, with mitochondrial dysfunction playing a key role in both sporadic and familial forms of the illness. Dysfunction of the mitochondria is caused by bioenergetic defects, mutations in mitochondrial DNA, nuclear DNA gene mutations linked to mitochondria, and changes in dynamics of the mitochondria such fusion or fission, changes in size and morphology, alterations in trafficking or transport, altered movement of mitochondria, impairment of transcription, and the presence of mutated proteins associated with mitochondria. ${ }^{11}$

Mitochondrial dysfunction is now recognised as an important determinant of dopaminergic neuronal susceptibility in Parkinson's disease, and it is seen in both familial and sporadic cases, as well as toxin-induced Parkinsonism. Recently, the mechanisms by which PDassociated mitochondrial proteins phosphatase and tensin homolog deleted on chromosome 10 (PTEN)-induced putative kinase-1 (PINK1) and parkin function induced neurodegeneration have been identified. Furthermore, there is growing evidence that other PDassociated proteins, including a-synuclein (a-syn) and leucine-rich repeat kinase 2 (LRRK2), are involved in mitochondrial dysfunction in hereditary instances of $\mathrm{PD}$, with the potential for a considerable functional overlap with sporadic disease. ${ }^{12}$ Pathogenic mutations in critical genes such as PARK7 (encoding DJ1), synuclein, parkin, PINK1, or LRRK2 cause PD. ${ }^{9} \mathrm{PD}$, like $\mathrm{AD}$, is characterized by mitochondrial dysfunction, which is mostly caused by mutations and random events that result in Parkin, encoded by the gene PARK2, or kinase PINK1, or both, losing function.

Parkin and PINK1 are involved in the mitophagy regulation of mitochondrial QC. ${ }^{3}$ Observations from experimental models and human PD provide strong evidence for disruptions in mitochondrial dynamics, bioenergetics defects, complex I inhibition of the electron transport chain (ETC), and increased reactive oxygen species (ROS). ${ }^{12,13}$ Parkin has a protective impact because it can connect with the outer mitochondrial membrane and prevents mitochondrial swelling, inhibits cytochrome $\mathrm{C}$ release, caspase trigger, and neuronal death, all of which are inhibited by parkin gene mutations. ${ }^{14}$

\section{Huntington's disease}

Huntington's disease (HD) is a neurodegenerative condition caused by the loss of neurons in the striatum. The major clinical symptoms of HD are progressive physical impairment, cognitive decline, and mental disorder. Progressive motor disability, personality changes, psychiatric illness, and intellectual deterioration are all other symptoms of the condition. Expansion of the CAG repeats in exon 1 of the huntingtin gene, which encodes Huntingtin $(\mathrm{Htt})$ protein, causes this condition. Various cellular and molecular events are involved in this process. Mitochondria, being key organelle, play an important part in the majority of neurodegenerative diseases, including HD. ATP production, $\mathrm{Ca} 2+$ buffering, ROS production, and antioxidant activity are all important functions of mitochondria in neurons. Neurons, being high-demand energy cells, are intimately linked to mitochondria's operation, maintenance, and dynamics. Mitochondrial activities and dynamics are altered in most neurological diseases, which are associated with excessive ROS levels, limited ATP production, and apoptosis. In this disorder, the accumulation of mutant huntingtin $(\mathrm{mHtt})$ may cause mitochondrial malfunction. HD is a polyglutamineexpansion condition that mostly affects the striatum and cerebral cortex. The mitochondrial malfunction has been identified as an early pathogenic mechanism outlining the selective neurodegeneration that occur in HD. The role of mitochondria in eukaryotic cells (e.g., neurons) have largely diverged from the classically attributed cell power source. Indeed, mitochondria are dynamic organelles that fragment and fuse to achieve maximum bioenergetic performance, they regulate intracellular calcium homeostasis through the interaction with the endoplasmic reticulum, produce free radicals and participate in cell death processes. ${ }^{15}$ Pathologically, the striatum loses medium spiny neurons preferentially and progressively, as well as cortical atrophy and there is degeneration of other brain regions later in the disease. ${ }^{16}$

\section{Concluding remarks}

Impaired mitochondrial dynamics, oxidative stress and mutations in the mitochondrial DNA underlie the major downstream processes of mitochondrial dysfunction that lead to a vast spectrum of neurodegenerative diseases. Mitochondrial dysfunction results in depletion of ATP which ultimately affects the enzyme complexes involved in ETC. Targeting energy metabolism in mitochondria, free radical production or disease related protein interaction with mitochondria lead to the preservation of mitochondrial functioning which is emerging as one of the best approaches to treat neurodegenerative diseases. Thus, apart from looking 
neurodegenerative diseases on the whole, a detailed understanding of the functioning of mitochondria and their role in degeneration would pave new options for the therapy of age-related neurodegenerative diseases.

\section{Funding}

None.

\section{Acknowledgments}

RK thankfully acknowledge AICTE New Delhi for GPAT Scholarship of M.Pharm. All authors thankfully acknowledge DPSRU, Govt. of NCT, New Delhi for providing needful infrastructure and research facilities.

\section{Conflicts of interest}

The authors declare that there is no conflict of interest.

\section{Supplementary materials}

None.

\section{References}

1. Bhatti JS, Bhatti GK, Reddy PH. Mitochondrial dysfunction and oxidative stress in metabolic disorders - A step towards mitochondria based therapeutic strategies. Biochim Biophys Acta Mol Basis Dis. 2017;1863(5):1066-1077.

2. Norat P, Soldozy S, Sokolowski JD, et al. Mitochondrial dysfunction in neurological disorders: Exploring mitochondrial transplantation. NPJ Regen Med. 2020;5(1):22.

3. Sorrentino V, Menzies KJ, Auwerx J. Repairing Mitochondrial Dysfunction in Disease. Anпи Rev Pharmacol Toxicol. 2018;58:353-389.

4. Srinivasan S, Guha M, Kashina A, et al. Mitochondrial dysfunction and mitochondrial dynamics-The cancer connection. Biochim Biophys Acta Bioenerg. 2017;1858(8):602-614.
5. Wu Y, Chen M, Jiang J. Mitochondrial dysfunction in neurodegenerative diseases and drug targets via apoptotic signaling. Mitochondrion. 2019;49:35-45.

6. Lin MT, Beal MF. Mitochondrial dysfunction and oxidative stress in neurodegenerative diseases. Nature. 2006;443(7113):787-795.

7. Johri A, Beal MF. Mitochondrial dysfunction in neurodegenerative diseases. J Pharmacol Exp Ther. 2012;342(3):619-630.

8. Shoshan-Barmatz V, Nahon-Crystal E, Shteinfer-Kuzmine A, et al. VDAC1, mitochondrial dysfunction, and Alzheimer's disease. Pharmacol Res. 2018;131:87-101.

9. Wang W, Zhao F, Ma X, et al. Mitochondria dysfunction in the pathogenesis of Alzheimer's disease: recent advances. Mol Neurodegener 2020;15(1):30.

10. Cadonic C, Sabbir MG, Albensi BC. Mechanisms of Mitochondrial Dysfunction in Alzheimer's Disease. Mol Neurobiol. 2016;53(9):6078-6090.

11. Bose A, Beal MF. Mitochondrial dysfunction in Parkinson's disease. $J$ Neurochem. 2016;139 Suppl 1:216-231.

12. Ryan BJ, Hoek S, Fon EA, et al. Mitochondrial dysfunction and mitophagy in Parkinson's: from familial to sporadic disease. Trends Biochem Sci. 2015;40(4):200-210.

13. Winklhofer KF, Haass C. Mitochondrial dysfunction in Parkinson's disease. Biochim Biophys Acta. 2010;1802(1):29-44.

14. Darios F, Corti O, Lucking CB, et al. Parkin prevents mitochondrial swelling and cytochrome $\mathrm{c}$ release in mitochondria-dependent cell death. Hum Mol Genet. 2003;12(5):517-526.

15. Carmo C, Naia L, Lopes C, et al. Mitochondrial Dysfunction in Huntington's Disease. Adv Exp Med Biol. 2018;1049:59-83.

16. Johri A, Beal MF. Antioxidants in Huntington's disease. Biochim Biophys Acta. 2012;1822(5):664-674. 